\title{
Article \\ Qualitative Investigation of Hamiltonian Systems by Application of Skew-Symmetric Differential Forms
}

\author{
Ludmila Petrova (D) \\ Faculty of Computational Mathematics and Cybernetic, Moscow State University, Moscow 119234, Russia; \\ ptr@cs.msu.ru
}

check for

updates

Citation: Petrova, L. Qualitative Investigation of Hamiltonian Systems by Application of Skew-Symmetric Differential Forms. Symmetry 2021, 13, 25. https://dx.doi.org/10.3390/ sym 13010025

Received: 31 October 2020

Accepted: 22 December 2020

Published: 25 December 2020

Publisher's Note: MDPI stays neutral with regard to jurisdictional claims in published maps and institutional affiliations.

Copyright: () 2020 by the author. Licensee MDPI, Basel, Switzerland. This article is an open access article distributed under the terms and conditions of the Creative Commons Attribution (CC BY) license (https: / / creativecommons.org/ licenses/by/4.0/).

\begin{abstract}
In the present paper, a role of Hamiltonian systems in mathematical and physical formalisms is considered with the help of skew-symmetric differential forms. In classical mechanics the Hamiltonian system is realized from the Euler-Lagrange equation as the integrability condition of the Euler-Lagrange equation and discloses specific features of Lagrange formalism. In the theory of differential equations, the Hamiltonian systems reveals canonical relations that define the integrability conditions of differential equations. The Hamiltonian systems, as a self-independent equations, are an example of dynamic systems that describe a behavior of dynamical systems in phase space. The connection of the Hamiltonian systems with differential equations and dynamical systems point to the fact that dynamical systems can be generated by differential equations. Under the investigation of Hamiltonian systems, in addition to exterior skew-symmetric differential forms it is suggested to use the skew-symmetric differential forms that are defined on a nonintegrable manifolds and possess a nontraditional mathematical apparatus, such as degenerate transformations and transitions from nonintegrable manifold to integral structures.
\end{abstract}

Keywords: Euler-Lagrange equation; Lagrangian and Hamiltonian manifolds; degenerate transformation; realization of Hamiltonian systems

\section{Introduction}

In the present paper, it is shown that a qualitative analysis of Hamiltonian systems with the help of skew-symmetric differential forms [1-3] enables one to describe a mechanism of Hamiltonian system implementation and uncover a role of invariant properties of the Hamiltonian systems in various branches of mathematics and mathematical physics.

Such possibitlities of studying the Hamiltonian systems with the help of skewsymmetric differential forms relates, firstly, to the fact that closed exterior skew-symmetric forms are invariant ones, and, secondly, there exist skew-symmetric forms [3] that are defined on nonintegrable manifolds and they generate closed exterior forms, namely, invariants. Such skew-symmetric forms disclose a mechanism of Hamiltonian systems generation.

In Section 2, it is shown that a difference between the Lagrange function, which is directly obtained from the Euler-Lagrange equation, and the Hamilton function that obeys the Hamiltonian system and is realized from the Euler-Lagrange equation, relates to the integrability of the Euler-Lagrange equation. The Lagrange function is defined on tangent (Lagrangian) manifold on which the Euler-Lagrange equation and, correspondingly, the Lagrange function, appear to be nonintegrable. Additionally, the Hamilton function is defined on structures (sections of bundle) of cotangent (phase, Hamiltonian) manifold, on which the Euler-Lagrange equation becomes integrable (the Lagrangian and Hamiltonian manifolds are not equivalent ones).

In Section 3, a connection of Hamiltonian systems with differential equations is shown. A partial differential equation of the first order that does not explicitly depend on the function desired is studied. The canonical relations, which have a form of Hamiltonian system, are integrability conditions of such an equation. This correspondence discloses 
properties of Hamiltonian systems, namely, a realization of Hamiltonian systems under degenerate transformations and properties of integral structures of cotangent manifold.

The realization of Hamiltonian system, i.e., the transition of a tangent manifold to a cotangent one, is an evolutionary process, namely, is a process of the emergence of physical structures.

In Section 4, the Hamilton-Jacobi equation is considered, on which the integrability conditions are identically imposed, namely, the Hamiltonian system is fulfilled. In this case, the Hamiltonian system is an example of dynamical system that enables one to investigate peculiarities of dynamical system.

The correspondence between Hamiltonian systems and field theory equations was considered. This relates to the fact that the Hamiltonian systems, as well as the field theory equations, are relations solutions to which there are not functions (as in differential equations), but differentials, i.e., closed exterior forms (invariants).

\section{Analysis of Hamiltonian Systems}

Hamiltonian systems arise in the problems of functional extremum that has wide application in quantum field theory and in the problems of classical mechanics at the basis of which such dynamic principles as the principle of minimal action, the principle of virtual motions, and so on lie.

The Hamiltonian system can be written in the form

$$
\frac{d q_{j}}{d t}=\frac{\partial H}{\partial p_{j}}, \quad \frac{d p_{j}}{d t}=-\frac{\partial H}{\partial q_{j}}
$$

The Hamilton function $H$ results from the Lagrange function $L$ under the Legendre transformation: $H\left(t, q_{j}, p_{j}\right)=p_{j} \dot{q}_{j}-L, p_{j}=\partial L / \partial \dot{q}_{j}$, which converts the Lagrange function $L\left(t, q_{j}, \dot{q}_{j}\right)$ defined on tangent manifold $\left\{q_{j}, \dot{q}_{j}\right\}$ into the Hamilton function $H\left(t, q_{j}, p_{j}\right)$ defined on cotangent manifold $\left\{q_{j}, p_{j}\right\}$.

The Hamiltonian system is connected with the Euler-Lagrange equation

$$
\frac{d}{d t} \partial L / \partial \dot{q}_{j}-\frac{\partial L}{\partial q_{j}}=0
$$

which specifies a curve that is an extremal of the functional.

The connection of Hamiltonian systems with the Euler-Lagrange equation can be traced by comparing the differential of Hamilton function $H(p, q, t)$ with the differential of the function $\left(p \dot{q}_{j}-L\right)$ (such comparison is presented in the paper [4]. However, in present case we shall focus our attention on some points of such comparison).

The total differential of the Hamilton function $H(p, q, t)$ is written in the form

$$
d H=\frac{\partial H}{\partial p_{j}} d p_{j}+\frac{\partial H}{\partial q_{j}} d q_{j}+\frac{\partial H}{\partial t} d t
$$

Additionally, the total differential of the Hamilton function expressed in terms of the Lagrange function $H=p_{j} \dot{q}_{j}-L$ has the form

$$
d H=\dot{q}_{j} d p_{j}-\frac{\partial L}{\partial q_{j}} d q_{j}-\frac{\partial L}{\partial t} d t
$$

These expressions will be identical under the condition

$$
\dot{q}_{j}=\frac{\partial H}{\partial p_{j}}, \quad \frac{\partial L}{\partial q_{j}}=-\frac{\partial H}{\partial q_{j}}, \quad \frac{\partial L}{\partial t}=-\frac{\partial H}{\partial t}
$$


From the Euler-Lagrange Equation (2), it follows that $\partial L / \partial q_{j}=\dot{p}_{j}$. Replacing in the second relation (3) the $\partial L / \partial q_{j}$ by $\dot{p}_{j}$, we obtain

$$
\frac{d p_{j}}{d t}=-\frac{\partial H}{\partial q_{j}}
$$

which corresponds to the second relation of Hamiltonian system. That is, the second relation of Hamiltonian system is just the Euler-Lagrange equation.

However, from relations (3), one can see that, changing from the Lagrange function to the Hamilton function in addition to the relation corresponding to the Euler-Lagrange equation, one more relation arises; namely, the first relation (3) that corresponds to the first relation for Hamiltonian system (1) and it is not connected with the Euler-Lagrange equation.

Thus, one can see that the Hamiltonian system contains an additional relation that is not directly connected with the Euler-Lagrange equation.

What is a physical meaning of additional relation.

The Euler-Lagrange equation has been obtained from the maximum condition of the action functional $S$ :

$$
\delta S=0
$$

where

$$
S=\int L d t
$$

In the actual case, when forces are nonpotential or couplings are nonholonomic, the quantity $\delta S$ is not a closed exterior form (the invariant), which is, $d \delta S \neq 0$. This points to the fact that the Euler-Lagrange equation appears to be nonintegrable on specified space. (It should be noted that functionals, such as wave function, the action functional, Pointing's vector, Einstein's tensor, and other [5], specify a variation of the system considered. This is connected with conservation laws.).

The condition (4) is satisfied along trajectories and this corresponds to conservation law for energy. However, the state of the system described also depends on the conservation law for momentum (and the conservation laws for angular momentum and mass). The peculiarity consists in the fact that the conservation law for momentum, which governs interactions between the trajectories, in a general case, without additional conditions, does not commutate with law for energy and, hence, the condition of invariance is not satisfied, i.e., $d \delta S \neq 0$.

However, the Hamiltonian system is obtained from the condition $d \delta S=0$, which is a closure condition for the form $\delta S$. This means that a closed exterior form has been realized, namely, a differential. However, it realizes discretely on the pseudostructures of cotangent space. The first relation of Hamiltonian systems describes such pseudostructures.

As it was noted, the Euler-Lagrange equation on tangent space appears to be nonintegrable, since $\delta S$ is not a closed form. The realization of Hamiltonian system, namely, the realization of pseudostructures (the sections of bundle) of cotangent space with closed form, is a realization of integrability conditions for the Euler-Lagrange equation.

The transition of the Euler-Lagrange equation from the tangent space, where the form is unclose (i.e., is not a differential) to cotangent space with closed form (which is a differential), is a degenerate transformation; namely, a transformation that does not conserve a differential. Such a transition is achieved with the help of the Legendre transformation being a degenerate transformation.

Here, it should be noted that the Legendre transformation is fulfilled under discrete realization of additional conditions that are conditioned by any degrees of freedom (the attention to this will be called in the next Section).

Because the Legendre transformation is discretely fulfilled, it can only be realized in closed inexact form, namely, the exterior form being closed only to a certain structure (a pseudostructure with respect to its metric properties) that is described by a dual form. 
The first relation of system (3) entered into the Hamiltonian system defines a pseudostructure on which a closed inexact exterior form is defined. This points to the integrability of the Hamilton system.

It can be noted that the closed inexact exterior form (a conservative quantity) and relevant dual form, which describe pseudostructure, made up a differential-geometrical structure that describes a physical structure (a structure with conservative quantity) on which conservation laws are obeyed. The solutions to Hamilton systems describe such structures.

(Here, the following may be pointed out. The Hamiltonian system is an example of a dynamic system. The fact that this system is generated by the Euler-Lagrange equation, i.e., by a differential equation, leads to a statement that dynamical systems can be generated by differential equations, i.e., by the equations that describe any processes. This will be considered in the next section.)

Let us consider one more peculiarity of Hamiltonian systems.

As it was already pointed out above, the Hamiltonian system was obtained with the help of degenerate transformation. However, on the other hand, the Hamiltonian system is a canonical relations which execute a nondegenerate transformation.

Such a peculiarity of Hamiltonian systems discloses a connection between degenerate and nondegenerate transformations. The degenerate transformation is a transition from tangent space $\left(q_{j}, \dot{q}_{j}\right)$ to cotangent manifold $\left(q_{j}, p_{j}\right)$. Additionally, the nondegenerate transformation is a transition in cotangent space from some pseudostructure (phase trajectory) $\left(q_{j}, p_{j}\right)$ to another pseudostructure $\left(Q_{j}, P_{j}\right)$. (The formula of canonical transformation can be written as $p_{j} d q_{j}=P_{j} d Q_{j}+d W$, where $W$ is the generating function).

The transition from tangent space to cotangent one under degenerate transformation when the closed exterior form is realized describes an origination of invariant structure. Additionally, the nondegenerate transformation (with the help of canonical relations) is a transition from one invariant structure to another invariant structure. This demonstrates the connection between the degenerate and nondegenerate transformations.

One can see that such a connection describes a mechanism of evolutionary processes.

\section{Connection of Hamiltonian Systems with the Equations of Mathematical Physics}

The Hamiltonian systems, as well as the field theory equations, are relations, rather then differential equations. A specific feature of such relations consists in the fact, as opposed to differential equations where solutions are functions, solutions to relations are differentials, namely, closed exterior forms that are invariants. Such relations describe physical structures, since the closed inexact exterior forms, which are conservative quantities, and relevant dual forms describe structures on which conservation laws are fulfilled. (In paper [6] demonstrated that closed exterior forms and the corresponding dual forms of the zero and first degree are solutions to the equations of Hamilton formalism; the electromagnetic field equations are based on closed exterior forms and its corresponding dual form of second degree; the gravitational field is based on closed exterior and dual forms of third degree.).

Such relations are obtained from the mathematical physics equations when studying the integrability of equations (that depends on the consistency of the derivatives of the described functions with respect to various variables and on the consistency of equations if the mathematical physics equations are a set of equations).

The Hamiltonian system results when studying the integrability of partial differential equation of the first order that does not explicitly depend on the function desired and it is resolved with respect to some variables, for example, the time $t$. That is, the equation has the form

$$
\frac{\partial u}{\partial t}+E\left(t, x^{j}, p_{j}\right)=0, \quad p_{j}=\frac{\partial u}{\partial x^{j}}
$$

The differential equation appears to be integrable if the derivatives of this equation made up a differential. This is true when the skew-symmetric differential form $\theta=p_{j} d x^{j}$ 
(the summation over repeated indices is implied) is a closed exterior form (the differential of skew-symmetric form equals zero), i.e., it is a differential.

In the general case, from Equation (5), it does not follow (explicitly) that the derivatives $p_{j}=\partial u / \partial x^{j}$, which obey to the Equation (and given boundary or initial conditions of the problem), made up a differential.

It turns out that, without additional conditions, the differential equation is nonintegrable on original coordinate space. On original coordinate space, the solution to differential equation is not a function, since the derivatives of this solution do not make up a differential.

In order to obtain a solution that is a function (i.e., the derivatives of this solution compose a differential), it is necessary to add a closure condition for the form $\theta=p_{j} d x^{j}$ and for corresponding dual form (in the present case, the functional $\frac{\partial u}{\partial t}+E\left(t, x^{j}, p_{j}\right)$ plays a role of a form dual to $\theta$ ):

$$
\left\{\begin{array}{l}
d\left(\frac{\partial u}{\partial t}+E\left(t, x^{j}, p_{j}\right)\right)=0 \\
d\left(p_{j} d x^{j}\right)=0
\end{array}\right.
$$

The dual form that corresponds to exterior differential form defines a structure, on which the closed inexact exterior form is defined.

In order to expand the differentials, one obtains a set of homogeneous equations with respect to $d x^{j}$ and $d p_{j}$ (in the $2 n$-dimensional cotangent space):

$$
\left\{\begin{array}{l}
\left(\frac{\partial E}{\partial x^{j}}\right) d x^{j}+\left(\frac{\partial E}{\partial p_{j}}\right) d p_{j}=0 \\
d p_{j} d x^{j}-d x^{j} d p_{j}=0
\end{array}\right.
$$

The solvability conditions for this system (vanishing the determinant that is composed of coefficients at $d x^{j}, d p_{j}$ ) have the form:

$$
\frac{d x^{j}}{\partial E / \partial p_{j}}=\frac{-d p_{j}}{\partial E / \partial x^{j}}=d t
$$

and can be reduced to the form

$$
\frac{d x^{j}}{d t}=\frac{\partial E}{\partial p_{j}}, \quad \frac{d p_{j}}{d t}=-\frac{\partial E}{\partial x^{j}}
$$

These are characteristic relations for Equation (5), which are conditions of integrability of this equation.

The canonical relations have just such a form, as is well known.

Characteristic relations determine an integrable structure on which the derivatives of Equation (5) $p_{1}=\partial u / \partial t, p_{j}=\partial u / \partial x^{j}$ made up a differential

$$
\left(-E d t+p_{j} d x^{j}\right)_{\pi}=d_{\pi} u
$$

namely, a closed inexact exterior form on the integrable structure that is a dual form. This points to the emergence of a physical structure that corresponds to conservation law, namely, a structure with conservative quantity (closed exterior form).

The solution to Equation (5) corresponding to such derivatives will be a discrete function.

One can see the correspondence between relations (8) (which are an example of canonical relations) and the Hamiltonian system. This correspondence enables one to disclose the peculiarities of Hamiltonian systems and its solutions.

\section{Peculiarities of Hamiltonian Systems}

It has been shown that Equation (5) only becomes integrable if the supplementary conditions, namely, the canonical relations (8), are realized. 
As it is known, for a description of integrable systems, the equation that obeys integrability conditions has been obtained. The Hamilton-Jacobi equation is such an equation.

Equations of such type include the equation

$$
\frac{\partial S}{\partial t}+H\left(t, q_{j}, \frac{\partial S}{\partial q_{j}}\right)=0, \quad \frac{\partial S}{\partial q_{j}}=p_{j}
$$

where $S$ is the action functional.

Corresponding characteristic relations for Equation (9) have the form

$$
\frac{d q_{j}}{d t}=\frac{\partial H}{\partial p_{j}}, \quad \frac{d p_{j}}{d t}=-\frac{\partial H}{\partial q_{j}}
$$

that is, they are a Hamiltonian system.

Here, it should be emphasized that, in spite of the fact that Equations (5) and (9) have the same form, they significantly differ from one another.

The distinction consists in the fact that Equation (5) is defined on tangent space, whereas Equation (9) does so on cotangent space.

Equation (5) is nonintegrable on original space, as has been shown. The drivatives of the solution do not make up a differential. The solution is not a function (but it has a physical meaning). The integrability (local) is possible if additional conditions (conditions of degenerate transformation), such as vanishing the determinant (that may be caused by any degrees of freedom) are realized.

Under degenerate transformation, the transition from tangent manifold to the cotangent one takes place. This occurs discretely when the conditions of degenerate transformation are fulfilled and the integrable structures (bundles of cotangent manifold) with a discrete function are realized, as it was shown.

That is, the solutions to Equation (5) possess a duality. They are obtained on original coordinate space as well as on integrable structures. A connection between these solutions and transitions from the solution on coordinate space to the solutions on integrable structures describe the mechanism of evolutionary processes that proceed in the system under consideration.

As opposed to Equation (5), Equation (9) is integrable. This equation obeys a Hamiltonian system. Such an equation can only have a solution on structures of cotangent space that are defined by the solution of Hamiltonian system.

Compatibility between Equations (5) and (9) shows that the Hamiltonian system may be realized from differential equation that describes any processes. The Euler-Lagrange equation is such an equation, as shown in Section 2.

From the analysis of Equation (5), one can see that the realization from differential equation of Hamiltonian system, as a canonic relation describes the evolutionary process of physical structures emergence. Additionally, Hamiltonian systems, as independent equations, are dynamic systems that describe only possible structures.

\section{Conclusions}

The investigation of Hamiltonian systems with the help of skew-symmetric differential forms reveals a unique role of Hamiltonian systems in various mathematical and physical formalisms.

In classical mechanics, the Hamiltonian system, as a integrability condition of the Euler-Lagrange equation, discloses a nonequivalent correspondence between Lagrange and Hamilton descriptions of the state of mechanical systems under consideration.

In the theory of differential equations, the Hamiltonian system as a canonical relation is a integrability condition of differential equations.

There is a correspondence between Hamiltonian systems and field theory equations. Hamiltonian systems, as well as the field theory equations, are relations (whose solutions 
are differentials) rather then differential equations (whose solutions are functions). It can be noted that, in the field theory, the Hamiltonian enters into the Schrodinger equation.

The Hamiltonian system, as an example of dynamical system, points out to a possible connection between dynamical systems and differential equations (as generators of dynamical systems).

In topology, the Hamiltonian system describes a structure of cotangent (covariant) space. In the theory of transformations, the Hamiltonian system (as a canonical relations) describes nondegenerate transformations (transformations that save a differential), which, in particular, are field theory transformations.

Funding: This research received no external funding.

Institutional Review Board Statement: Not applicable.

Informed Consent Statement: Not applicable.

Data Availability Statement: Data sharing not applicable.

Conflicts of Interest: The author declare no conflict of interest.

\section{References}

1. Cartan, E. Les Systemes Differentials Exterieus ef Leurs Application Geometriques; Exposes de Geometrie: Paris, France, 1945.

2. Schutz, B.F. Geometrical Methods of Mathematical Physics; Cambrige University Press: Cambrige, UK, 1982.

3. Petrova, L.I. Role of Skew-Symmetric Differential Forms in Mathematics. arXiv 2010, arXiv:1007.4757.

4. Arnold, V.I. Mathematical Methods of Classical Mechanics; Springer: New York, NY, USA, 1997.

5. Petrova, L.I. Physical meaning and a duality of concepts of wave function, action functional, entropy, the Pointing vector, the Einstein tensor. J. Math. Res. 2012, 4. [CrossRef]

6. Petrova, L.I. Basic Principles of the Field Theory: Connection of the Field-Theory Equations with the Equations of Mathematical Physics. Int. J. Adv. Res. Phys. Sci. (IJARPS) 2017, 4, 35-47. 